\title{
INFLUÊNCIA DE CATALISADORES ZEOLÍTICOS NOS PRODUTOS DE CRAQUEAMENTO DO ÓLEO DE SOJA
}

\author{
E. Y. EMORI ${ }^{1}$, F. H. HIRASHIMA ${ }^{1}$, C. H. ZANDONAI ${ }^{2}$, N. R. C. FERNANDES- \\ MACHADO $^{1}$ e M. H. N. OLSEN SCALIANTE ${ }^{1}$
}

${ }^{1}$ Universidade Estadual de Maringá, Departamento de Engenharia Química

${ }^{2}$ Universidade Estadual de Maringá, Centro de Tecnologia, Campus Regional de Umuarama

E-mail para contato: erickyukiemori@gmail.com

\begin{abstract}
RESUMO - O presente trabalho é dedicado a avaliação do craqueamento do óleo de soja na presença das zeólitas comerciais ZSM-5 e NaY. Os catalisadores foram calcinados $\left(500^{\circ} \mathrm{C}\right.$ por $\left.6 \mathrm{~h}\right)$, peneirados (mesh de $\left.0,417-0589\right)$, peletizados e caracterizados por Difração de Raios-X (DRX), análise textural e RMN de ${ }^{27} \mathrm{Al}$ e ${ }^{29} \mathrm{Si}$. Os testes de craqueamento foram realizados em temperatura moderada num reator tubular com fluxo de nitrogênio e hidrogênio a $42 \mathrm{~mL} / \mathrm{min}$. Os produtos foram avaliados a partir de cromatografia por espectro de massa e a gás, constatando que nas condições avaliadas os catalisadores utilizados promoveram maior formação de fração gasosa e a fração líquida obtida apresentou seletividade na faixa de gasolina, querosene e óleo diesel.
\end{abstract}

\section{INTRODUÇÃO}

A demanda por questões ambientais e o preço do petróleo abriu caminho para uma extensa área de pesquisa na produção de biocombustíveis a partir de óleos vegetais. Dentre os métodos desenvolvidos pode-se citar a transesterificação e o craqueamento termocatalítico a elevadas temperaturas, que produz combustível semelhante a frações de petróleo (hidrocarbonetos gasosos, gasolina, querosene e diesel).

Apesar da incorporação de óleos vegetais transesterificados com metanol ou etanol ser uma realidade no Brasil, tal tecnologia apresenta alguns problemas ainda não solucionados. Desta forma, o craqueamento catalítico de óleos vegetais é uma alternativa que deve ser considerada, pois são produzidos hidrocarbonetos da faixa do diesel de petróleo, com a vantagem de não modificar o funcionamento do motor e não ser de origem fóssil. Frações mais leves por ventura produzidas poderão ser incorporadas em outros combustíveis como gasolina e gás liquefeito do petróleo (GLP).

A tecnologia de craqueamento catalítico de frações de petróleo, como a nafta pesada, está plenamente desenvolvida (Corma et al., 2013), sendo uma das principais plantas em uma refinaria. O catalisador utilizado é uma mistura de vários componentes, sendo a zeólita $\mathrm{NaY}$ seu principal constituinte, cuja formulação varia em função das características da nafta a ser craqueada. Dentre todas as zeólitas já sintetizadas, a zeólita Y é a mais utilizada industrialmente, apresentando razões de silício e alumínio superior a 1,5, muito utilizada em sua forma ácida. Em reações tradicionais de craqueamento, a zeólita Y apresenta maior área 
superficial e grande quantidade de sítios ativos, essenciais para a atividade do catalisador (KULPRATHIPANJA, 2010).

A literatura aponta uma série de trabalhos utilizando materiais zeolíticos no craqueamento de óleos vegetais (Rahimi e Karimzadeh, 2011; Borges et al., 2011; Rabeharitsara et al., 2003; Scofield et al., 1998), porém, tais trabalhos baseiam-se no que atualmente é considerado um dos maiores desafios da ciência: o projeto e controle de sistemas químicos sobre múltiplas escalas. Nesse contexto, a catálise heterogênea tem se beneficiado de propriedades particulares desses materiais (Ocampo et al., 2010) e diferentes zeólitas com propriedades sensíveis ao seu tamanho são apontadas para governar a seletividade em diversas reações catalíticas (Yang et al., 2009) e, consequentemente, diversos materiais zeolíticos foram apontados como promissores catalisadores no craqueamento catalítico de óleos vegetais (Li et al., 2014), tais como ZSM-5/MCM-41 (Corma et al., 2013; Nam et al., 2011), HZSM-5 suportada em Ni e Mo (Cheng et al., 2014).

Desta forma, o presente trabalho tem como objetivo principal avaliar a influência do gás hidrogênio nos produtos de craqueamento catalítico do óleo de soja a partir da utilização das zeólitas NaY e ZSM-5.

\section{MATERIAIS E MÉTODOS}

\subsection{Preparo dos catalisadores}

O presente trabalho utilizou zeólitas comerciais $\mathrm{NaY}$ e ZMS-5 que foram calcinadas a $500{ }^{\circ} \mathrm{C}$ seguindo a metodologia proposta por Zandonai, (2014) na qual o processo se inicia a temperatura ambiente e tem sua temperatura elevada a partir de uma rampa de aquecimento de $2^{\circ} \mathrm{C} / \mathrm{min}$ até atingir $120^{\circ} \mathrm{C}$ permanecendo nesta temperatura por $1 \mathrm{~h}$ e depois $2^{\circ} \mathrm{C} / \mathrm{min}$ até $200^{\circ} \mathrm{C}$ ficando nessa temperatura por mais $1 \mathrm{~h}$ para finalmente a $2^{\circ} \mathrm{C} / \mathrm{min}$ até atingir temperatura final de $500^{\circ} \mathrm{C}$ na qual foi mantido por $6 \mathrm{~h}$. Após a calcinação, os catalisadores foram peneirados (mesh de 0,417 - 0589) e peletizados (compressão de $3000 \mathrm{kgf} / \mathrm{cm}^{2}$ ).

\subsection{Caracterização dos catalisadores}

Depois de preparadas, as zeólitas foram submetidas a análises de caracterização pelas seguintes técnicas:

Análise textural: realizada a partir de isotermas de adsorção de $\mathrm{N}_{2}$ a $77 \mathrm{~K}$ utilizando o equipamento QuantaChrome NOVA do Laboratório de Catálise DEQ/UEM.

Ressonância magnética nuclear (RMN): Tal análise foi realizada no Laboratório de Pesquisa e Extensão do Departamento de Química da UEM (DQI/UEM) em equipamento Mercury Plus 300, da Varian, equipado com sonda para a análise em sólidos CP/MAS $7 \mathrm{~mm}$. A operação ocorreu a 59,6132 MHz para o núcleo ${ }^{29} \mathrm{Si}$ e a $78,186 \mathrm{MHZ}$ para o núcleo ${ }^{27} \mathrm{Al}$ usando como referência o sinal do caulim a $-91,16 \mathrm{ppm}$.

Difração de raios- $X$ : para tal análise utilizou-se um difratômetro de Raios-X Bruker D8 Advance, 2teta de 5 a $85^{\circ}$, radiação $\mathrm{CuK \alpha}, 0,24 \%$ min na varredura, $40 \mathrm{kV}$ e $50 \mathrm{~mA}$ do Complexo de Centrais de Apoio à Pesquisa da UEM, COMCAP/UEM. Os picos observados foram comparados com os dados publicados pelo ICDD $\mathrm{PDF}_{2}$ (Philips). 


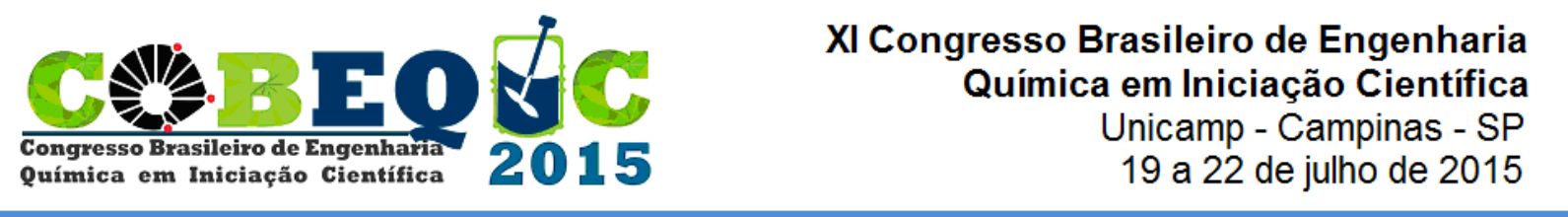

\subsection{Testes Catalíticos}

Os testes catalíticos foram conduzidos em microrreator de leito fixo conforme esquematizado na Figura 1. Utilizou-se óleo de soja bruto obtido da linha de produção, sem que tivesse passado pela degomagem cuja composição encontra-se disposta na Tabela 1 a seguir.

Tabela 1 - Composição do óleo de soja bruto.

\begin{tabular}{ccc}
\hline Ácidos orgânicos do óleo & Fórmula molecular & Concentração (\%) \\
\hline Ácido hexadecanoico & $\mathrm{C}_{16} \mathrm{H}_{32} \mathrm{O}_{2}$ & 12,93 \\
Ácido 9,12-octadecadienoico & $\mathrm{C}_{18} \mathrm{H}_{30} \mathrm{O}_{2}$ & 36,81 \\
Ácido 9-octadecenoico & $\mathrm{C}_{18} \mathrm{H}_{32} \mathrm{O}_{2}$ & 44,08 \\
Ácido 16-octadecenoico & $\mathrm{C}_{18} \mathrm{H}_{32} \mathrm{O}_{2}$ & 1,30 \\
Ácido octadecanoico & $\mathrm{C}_{18} \mathrm{H}_{36} \mathrm{O}_{2}$ & 4,87 \\
\hline
\end{tabular}

Figura 1 - Módulo experimental

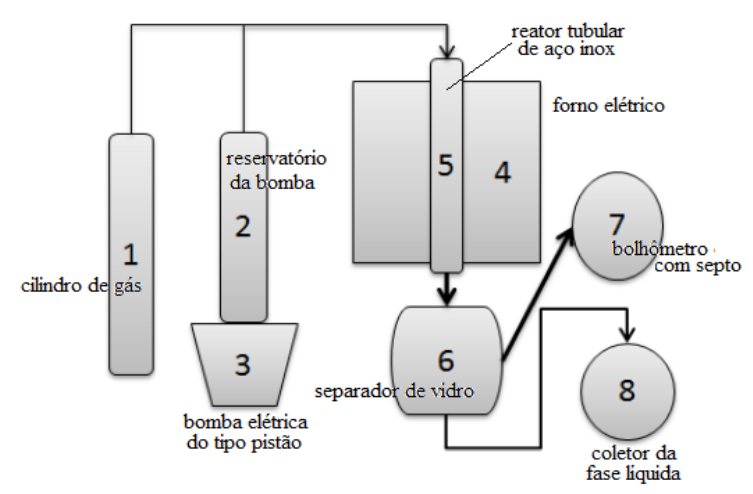

A ativação dos catalisadores foi realizada com a passagem de nitrogênio a $42 \mathrm{~mL} / \mathrm{min}$ por uma hora a $450^{\circ} \mathrm{C}$ (rampa de $30^{\circ} \mathrm{C} / \mathrm{min}$ ). Testes foram conduzidos também com $\mathrm{N}_{2}$ a fim de averiguar a influência do $\mathrm{H}_{2}$ na distribuição de produtos. Por meio de bomba elétrica, óleo de soja bruto foi conduzido ao reator numa vazão de $0,07 \mathrm{~g} / \mathrm{min}$. O tempo reacional foi de 45 min e os produtos foram separados por condensador de vidro.

\subsection{Análise dos Produtos}

Os produtos gasosos foram analisados por cromatografia gasosa em um cromatógrafo da marca Varian, modelo 1420, com detector de condutividade térmica (TCD), equipado com uma coluna Porapak Q (3,15 mm de diâmetro de $90 \mathrm{~cm}$ de comprimento). A identificação dos picos foi realizada com injeção de misturas gasosas com padrões de $\mathrm{CO}, \mathrm{CO}_{2}, \mathrm{CH}_{4}$, Etano, Eteno, Propano, Propeno, Butano, $\mathrm{N}_{2}$, Água e Isopentano.

Os produtos líquidos receberam pré-tratamento conforme metodologia descrita por Hartman e Lago (1973) que consiste de metilação por catálise ácida em que $2 \mathrm{~mL}$ de solução de $\mathrm{NaOH} 0,5 \mathrm{~mol} / \mathrm{L}$ em metanol foi adicionado à $100 \mathrm{mg}$ do produto líquido. Em seguida aquecida a $90^{\circ} \mathrm{C}$ por 5 min e após um rápido resfriamento em água corrente foi adicionado 
$3 \mathrm{~mL}$ de agente de esterificação $\left(33,33 \mathrm{~g}\right.$ de $\mathrm{NH}_{4} \mathrm{Cl}$ e $50 \mathrm{~mL}$ de $\mathrm{H}_{2} \mathrm{SO}_{4}$ em $1 \mathrm{~L}$ de $\left.\mathrm{CH}_{3} \mathrm{OH}\right)$. Aqueceu-se novamente a $90^{\circ} \mathrm{C}$ por $3 \mathrm{~min}$ e após, adicionou-se $2 \mathrm{~mL}$ de solução saturada de $\mathrm{NaCl}$ para separação de fases e $3 \mathrm{~mL}$ de isopentano e enviado para cromatografia líquida em cromatógrafo equipado com espectro de massa de coluna DB-5 com $30 \mathrm{~m}$ de comprimento, $0,25 \mathrm{~mm}$ de diâmetro e $0,25 \mu \mathrm{m}$ de fase estática. Os componentes foram identificados de acordo com a massa de seus íons na biblioteca do equipamento. O programa "Xcalibur" (“Thermo Electron”) foi empregado para a aquisição e processamento dos dados.

\section{RESULTADOS E DISCUSSÃO}

\subsection{Análise Textural: Fissisorção de Nitrogênio}

Ambos os catalisadores apresentaram uma pequena histerese nas isotermas, sendo eminentemente microporosos, característico de materiais zeolíticos. Valores apresentados na Tabela 2 foram utilizados a título de comparação, pois, não seria adequado o uso de ajuste pelo método BET para constante negativa. A zeólita $\mathrm{NaY}$ apresentou área específica superior á zeólita ZSM-5 e ambos os catalisadores apresentaram elevada área de microporos e um pequeno volume de mesoporos que é característico das zeólitas microporosas.

Tabela 2 - Distribuição das áreas específicas, constante BET e volume de poros.

\begin{tabular}{ccccccc}
\hline Amostra & $\begin{array}{c}\text { Área } \\
\text { Específica } \\
\left(\mathrm{m}^{2} / \mathrm{g}\right)\end{array}$ & $\begin{array}{c}\text { Área de } \\
\text { Microporos } \\
\left(\mathrm{m}^{2} / \mathrm{g}\right)\end{array}$ & $\begin{array}{c}\text { Área } \\
\text { Externa } \\
\left(\mathrm{m}^{2} / \mathrm{g}\right)\end{array}$ & $\begin{array}{c}\text { Constante } \\
\text { BET }\end{array}$ & $\begin{array}{c}\text { Volume de } \\
\text { microporos } \\
\left(\mathrm{cm}^{3} / \mathrm{g}\right)\end{array}$ & $\begin{array}{c}\text { Volume de } \\
\text { mesoporos } \\
\left(\mathrm{cm}^{3} / \mathrm{g}\right)\end{array}$ \\
\hline ZSM5 & 317,8 & 234,2 & 83,5 & $-52,75$ & 0,122 & 0,00146 \\
$\mathrm{NaY}$ & 656 & 637,72 & 18,08 & $-34,53$ & 0,329 & 0,0021 \\
\hline
\end{tabular}

\subsection{Difração de raios-X e Ressonância Magnética Nuclear}

De acordo com o apresentado por Treacy e Higgins, (2001) as análises de difração de raios-X confirmaram que as zeólitas ZSM-5 e NaY utilizadas apresentam cristalinidade característica de sua estrutura mesmo após o processo de calcinação.

As análises de ressonância magnética de ${ }^{29} \mathrm{Si}$ mostraram a diferença na razão entre $\mathrm{Si} / \mathrm{Al}$ dos catalisadores empregados. Como já esperado, a zeólita ZSM-5 apresentou uma razão $\mathrm{Si} / \mathrm{Al}$ de aproximadamente 19, afinal, a síntese dessa zeólita utiliza uma quantidade superior de silício. Diferentemente, a zeólita $\mathrm{NaY}$ apresentou razão Si/Al entre 1 e 2.

\subsection{Análise dos Produtos}

A Tabela 3 dispõe os produtos pelo número de carbonos formados nos testes catalíticos bem como o percentual de $\mathrm{CO}_{2}$ e $\mathrm{H}_{2} \mathrm{O}$, para o fluxo de $\mathrm{H}_{2}$ e $\mathrm{N}_{2}$. Verificou-se uma maior seletividade para gás carbônico e água para o craqueamento com fluxo de hidrogênio. Isso evidencia a interação do gás hidrogênio com o craqueamento, reagindo com o oxigênio presente nos ácidos orgânicos, removendo-o da molécula sobre forma de óxidos gasosos mais simples, como os citados acima. Esse processo é conhecido como hidrotratamento e ocorre não só com os átomos de oxigênio, mas com qualquer heteroátomo presente na cadeia (SOUZA, 2011). Observou-se uma diferença significativa na distribuição dos produtos 
formados o que evidencia a especificidade de cada zeólita utilizada como catalisador. A zeólita $\mathrm{NaY}$ demonstrou maior capacidade de craqueamento com maiores concentrações de hidrocarbonetos de cadeia curta, já que possui maior área superficial e maior volume de micro e mesoporos como visto em sua análise textural. Consequentemente houve maiores quantidades de reações para esta zeólita do que para a ZSM-5.

Tabela 3 - Percentual de produtos gasosos formados para cada zeólita com fluxo de $\mathrm{H}_{2}$ e $\mathrm{N}_{2}$.

\begin{tabular}{ccccc}
\hline Produtos & $\mathrm{ZSM}-5\left(\mathrm{H}_{2}\right)[\%]$ & $\mathrm{ZSM}-5\left(\mathrm{~N}_{2}\right)[\%]$ & $\mathrm{NaY}\left(\mathrm{H}_{2}\right)[\%]$ & $\mathrm{NaY}\left(\mathrm{N}_{2}\right)[\%]$ \\
\hline $\mathrm{C} 1$ & 3.36 & 4.38 & 24.76 & 33.06 \\
$\mathrm{C} 2$ & 12.33 & 8.51 & 22.62 & 14.02 \\
$\mathrm{C} 3$ & 44.21 & 41.29 & 8.15 & 9.23 \\
$\mathrm{C} 4$ & 22.21 & 25.44 & 2.55 & 27.18 \\
$\mathrm{C} 5$ & 1.82 & 16.56 & 2.23 & 11.08 \\
$\mathrm{H}_{2} \mathrm{O}$ & 2.48 & 2.32 & 14.76 & 0.00 \\
$\mathrm{CO}_{2} / \mathrm{CO}$ & 13.60 & 1.49 & 22.58 & 0.00 \\
\hline
\end{tabular}

A Tabela 4 apresenta a quantidade de carbonos nos hidrocarbonetos líquidos resultantes do craqueamento. Os produtos foram divididos de acordo com o número de carbonos sendo, também, quantificada a quantidade de ácidos orgânicos e radicais oxigenados presentes. Similarmente ao comportamento dos produtos gasosos notou-se que o gás $\mathrm{H}_{2}$ influencia no mecanismo de desoxigenação dos ácidos orgânicos, pois o craqueamento com o fluxo de $\mathrm{N}_{2}$ apresentou maiores quantidades de ácidos. Porém, a quantidade de radicais oxigenados demonstrou uma desoxigenação incompleta, principalmente com a zeólita $\mathrm{NaY}$, retirando apenas o oxigênio que liga-se a cadeia carbônica dos ácidos através da ligação $\pi$. Observou-se maior seletividade da zeólita ZSM-5 em relação aos produtos líquidos mais pesados. Isso se deve a grande capacidade de craqueamento desse catalisador que permitiu a aromatização das cadeias, detectadas pelo detector de espectro de massa. Essas moléculas apresentam maior estabilidade e pontos de ebulição altos, permanecendo por mais tempo nesse estado. A zeólita $\mathrm{NaY}$ apresentou grande quantidade de alcanos lineares, evidenciando baixa capacidade de craqueamento para moléculas muito grandes, com reduzidas quantidades de hidrogenações no mecanismo de reação.

Tabela 4 - Concentração dos produtos formados para cada zeólita em cada fluxo gasoso.

\begin{tabular}{ccccc}
\hline Produtos & ZSM-5 $\left(\mathrm{H}_{2}\right)[\%]$ & $\mathrm{ZSM}-5\left(\mathrm{~N}_{2}\right)[\%]$ & $\mathrm{NaY}\left(\mathrm{H}_{2}\right)[\%]$ & $\mathrm{NaY}\left(\mathrm{N}_{2}\right)[\%]$ \\
\hline C5-C8 & 19.32 & 77.24 & 3.41 & 6.63 \\
C8-C16 & 79.16 & 22.02 & 60.75 & 47.81 \\
Ácidos & 0.00 & 0.71 & 21.29 & 33.19 \\
Oxigenados & 1.52 & 0.03 & 14.56 & 13.85 \\
\hline
\end{tabular}

\section{CONCLUSÃO}

Para as condições de teste, observou-se que ambas as zeólitas atuam eficientemente como catalisadoras no craqueamento do óleo de soja, gerando produtos gasosos leves e produtos líquidos na faixa da gasolina, querosene e óleo diesel. O uso de NaY e ZSM-5 
favoreceu distinta distribuição de produtos e seletividade. A participação do gás hidrogênio foi constatada por meio da desoxigenação das moléculas sobre forma de água, $\mathrm{CO}_{2}$ e $\mathrm{CO}$.

\section{AGRADECIMENTOS}

Os autores agradecem ao DEQ/UEM e à Fundação Araucária pelo apoio financeiro.

\section{REFERÊNCIAS}

BORGES, C., De GIOVANNI, M. Y. G., CABRAL, G., PERGHER, S. B. C., FERNANDES-MACHADO, N. R. C., 2011, Craqueamento catalítico do n-heptano usando a zeólita MCM22 com diferentes razões Si/Al, In: $16^{\circ}$ Congresso Brasileiro de Catálise, Campos do Jordão, SP.

CHENG, J., HUANG, T. L., JUNHU, Z., CEN, K., Optimizing catalysis conditions to decrease aromatic hydrocarbons and increase alkanes for improving jet biofuel quality. Bio Technol, v. 158, p. 378-382, 2014.

CORMA, A.; MENGUAL, J.; MIGUEL, P. J. IM-5 zeolite for steam catalytic cracking of naphtha to produce propene and ethane. An alternative to ZSM-5 zeolite. App. Catal. A: General, v. 460 - 461, p. 106 - 115, 2013.

HARTMAN, L.; LAGO, R. C. A. Rapid preparation of fatty acid methyl from lipids. Laboratory Practice, v. 22, n. 3, p. 475-473, 1973.

KULPRATHIPANJA, S. Zeolites in Industrial Separation and Catalysis, Wiley-VCH Verlag GmbH \& Co. KGaA; Weinheim, 2010.

LI, L., QUAN, K., XU, J., LIU, F., LIU, S., YU, S., XIE, C., ZHANG, B., GE, X., Liquid hydrocarbon fuels from catalytic cracking of rubber seed oil using USY as catalyst. Fuel, n.123, p.189-193, 2014.

NAM, L. T. H., VINH, T. Q., LOAN, N. T. T., THO, V. D. S., YANG, X., SU, B., Preparation of bio-fuels by catalytic cracking reaction of vegetable oil sludge. Fuel, n. 90, p. $1069-1075,2011$.

OCAMPO, F., CUNHA, J. A., DE LIMA SANTOS, M. R., TESSONNIER, J. P., PEREIRA, M. M., LOUIS, B., Synthesis of zeolite crystals with unusual morphology: Application in acid catalysis. Appl. Catal. A: General, n. 390, p. 102-109, 2010.

RABEHARITSARA, A., CERQUEIRA, H.S., MAGNOUX, P., GUISNET, M., COSTA, A. F., SOUZA-AGUIAR, E. F., Transformation of methylcyclohexane on an FCC catalyst. Braz. J. Chem. Eng., v. 20, n. 2, 2003.

RAHIMI, N.; KARIMZADEH, R. Catalytic cracking of hydrocarbons over modified ZSM-5 zeolites to produce ligh olefins: A review. App. Catal. A: General, v. 398, p. 1-17, 2011.

SCOFIELD, C.F., BENAZZI, E., CAUFFRIEZ, H. e MARCILLY, C. Metylcyclohexane conversion to light olefins, Braz. J. Chem. Eng., v.15, n. 2, 1998.

SOUZA, M. J. B. Beneficiamento da fração C5+ do Pólo de Guamaré a partir de reações de craqueamento catalítico sobre zeólitas ácidas. Universidade Federal do Rio Grande do Norte, 2011.

TREACY, .M.J.; HIGGINS, J. B. Collection of Simulated XRD Powder Patterns for Zeolites. Amsterdam: Elsevier, 2001. 